Pathologe $2011 \cdot$ [ [Suppl 2] 32:156

DOI 10.1007/s00292-011-1519-y

Online publiziert: 15. September 2011

(c) Springer-Verlag 2011
M. Dietel

Institut für Pathologie, Charité-Universitätsmedizin Berlin, Campus Charité Mitte, Berlin

\title{
Eröffnungsrede des Vorsitzenden der Deutschen Gesellschaft für Pathologie e.V.
}

Sehr verehrte Mitglieder der Deutschen Gesellschaft für Pathologie,

sehr verehrte Gäste, meine sehr verehrten Damen und Herren,

hiermit möchte ich Sie sehr herzlich zur Eröffnungsveranstaltung der 95. Jahrestagung der Deutschen Gesellschaft für Pathologie hier in Leipzig begrüßen. Gerade habe ich vom Tagungspräsidenten Herrn Prof. Wittekind erfahren, dass unsere Gesellschaft das letzte Mal vor 102 Jahren hier getagt hat, also im Jahre 1909. Somit war es wirklich dringend an der Zeit, diese Stadt und ihre Pathologie, die zu den wichtigen Zentren unseres Faches gehört, wieder einmal um die Durchführung der Jahrestagung unserer Gesellschaft zu bitten. Hier und jetzt ist es mir ein besonderes Anliegen, Ihnen, Herr Wittekind, und Ihren Mitarbeiterinnen und Mitarbeitern sehr herzlich dafür zu danken, dass sie sich dieser organisatorischen Mühe unterzogen und ein exzellentes Programm zusammengestellt haben. Darin spiegeln sich nach meiner Auffassung die Entwicklungen und Herausforderungen für die kommenden 10 Jahre umfänglich wider.

Der in meinen Augen zentrale Punkt besteht darin, dass wir Pathologen im Rahmen der prädiktiven (Immun-/Molekular-)Pathologie immer näher an die Klinik heranrücken und zusätzlich zu unserer Kernkompetenz der histologisch basierten Diagnose immer häufiger die Therapieentscheidungen direkt beeinflussen. Daraus ergibt sich zwangsweise, dass wir dringend darauf achten müssen, unsere Qualitätssicherungsmechanismen weiter zu schärfen. Die Pathologen müssen in allen ihren Aufgabengebieten flächendeckend reproduzierbare Ergebnisse ab- liefern können. Dass es daran mangelt, wäre zu hart formuliert, dass wir aber daran weiter intensiv arbeiten müssen, ist sicher nicht verkehrt.

Der zweite wichtige Themenkreis betrifft den Nachwuchs, um den wir uns intensiver kümmern müssen. Dies stellt eine Herausforderung für uns Pathologen insgesamt dar, die wir in Zusammenarbeit mit dem Bundesverband Deutscher Pathologen - ich darf Herrn Prof. Schlake als Vertreter des Bundesverbandes sehr herzlich begrüßen - meistern müssen. Mit mehreren Veranstaltungen im Hauptprogramm dieses Kongresses wird das Thema der systematischen Nachwuchsförderung aufgenommen und intensiv besprochen. Der Vorstand ist Herrn Wittekind und Herrn Schirmacher als Beauftragter für den Nachwuchs dafür besonders dankbar.

Neben diesen zwei strukturell bedeutsamen Punkten ist der wissenschaftliche Inhalt der Jahrestagung, der durch das Thema „Vorläuferläsionen“ von Herrn Wittekind vorgegeben wurde, eine Fragestellung, die die Zukunft der Pathologie entscheidend mit prägen wird. Wir werden in der täglichen Diagnostik immer häufiger mit Vorläuferläsionen konfrontiert werden und müssen dann eine qualifizierte Aussage machen. Die Kliniker werden uns fragen: Was wird daraus, was muss ich tun? Auch hier kommt wieder die prädiktive Pathologie ins Spiel. Die Beantwortung dieser Fragen ist schwierig und bei zahlreichen Veränderungen noch ungeklärt. Es ergibt sich also ein wissenschaftliches Aufgabenfeld, das aktiv und progressiv bearbeitet werden muss.

Die genannten Fragestellungen, die hier von mir nur kurz skizziert werden konnten, sind in dem Programm von Ihnen, lieber Herr Wittekind, exzellent eingearbeitet worden, und wir danken Ihnen sehr dafür.

In diesem Sinne möchte ich hiermit die 95. Jahrestagung der Deutschen Gesellschaft für Pathologie eröffnen und darf als Nachredner den Gastgeber auf das Podium bitten.

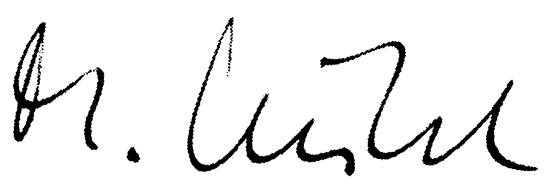

M. Dietel, Berlin

\section{Korrespondenzadresse \\ Prof. Dr. Dr. h.c. M. Dietel \\ Institut für Pathologie, \\ Charité-Universitätsmedizin Berlin \\ Campus Charité Mitte \\ Charitéplatz 1, 10117 Berlin \\ manfred.dietel@charite.de}

Interessenkonflikt. Der korrespondierende Autor gibt an, dass kein Interessenkonflikt besteht.

The supplement this article is part of is not sponsored by the industry. 